\title{
Comparación entre el aspecto endoscópico del cardias, hallazgos manométricos y pH metría de 24 horas en pacientes con síntomas de reflujo gastroesofágico crónico
}

\author{
Manlio Falavigna, Attila Csendes J, Ana Henríquez Da, \\ Rafael Luengas $\mathrm{T}$. \\ Comparison of the endoscopic \\ aspect of the cardia, manometry and \\ 24 hours $\mathrm{pH}$ measurement in \\ patients with chronic \\ gastroesophageal reflux
} \begin{abstract}
related with the manometric competence of the lower esophageal sphincter (LES) and with pathological gastroesophageal reflux. Aim: To compare the endoscopic aspect of the cardia with the results from esophageal manometry and $24 \mathrm{~h}$ esophageal $\mathrm{pH}$ monitoring. Materials and Methods: The cardia of a group of 150 patients (aged 19 to 72 years, 89 women) with gastroesophageal reflux symptoms was classified as normal or open, during upper gastrointestinal endoscopy. All subjects were subjected to a manometric study to measure LES pressure and determine LES incompetence and to a $24 \mathrm{~h}$ esophageal $\mathrm{pH}$ measurement. Results: LES pressure was $14.7 \pm 6.2$ and $8.7 \pm 4.2 \mathrm{mmHg}$ in patients with normal and open cardia, respectvively ( $p<0.001$ ). Likewise, 43 and $79 \%$ of patients with normal and open cardia had an incompetent LES, respectively ( $p<0.001$ ). Pathological acid reflux was present in 43 and $71 \%$ of patients with normal or open cardia, respectively $(\mathrm{p}<0.001)$. Conclusions: The endoscopic appearance of the cardia can identify patients with pathological gastroesophageal reflux (Rev Méd Chile 2006; 134: 187-92).
\end{abstract}

Background: The endoscopic view of the gastric cardia could be

(Key w ords: Esophageal pH monitoring; Gastroesophageal reflux; Manometry)

Recibido el 19 de mayo, 2005. Aceptado el 11 de agosto, 2005.

Departamento de Cirugía, Hospital Clínico de la Universidad de Chile. Santiago de Chile. aTecnólogo Médico.

Correspondencia a: Dr. Attila Csendes. Departamento de Cirugía, Hospital Clínico Universidad de Chile, Santiago, Chile. Fono: 56-2-7774387. Fax: 56-2-7775043.

E-mail: acsendes@machi.med.uchile.cl 
$\mathrm{V}$ arios autores han descrito en pacientes con reflujo gastroesofágico (GE) crónico una alteración anatómica de la unión gastroesofágica 0 cardias, que favorece la presencia y la gravedad de la sintomatología ${ }^{1,2}$. Este hallazgo anatómico patológico corresponde a una dilatación progresiva del cardias y ha sido denominada por algunos autores como patulous cardia ${ }^{1,2}$ o dilated cardia $^{3,4}$. En 1981 demostramos la diferencia del perímetro del cardias en sujetos controles comparados con pacientes con esofagitis ${ }^{5}$. Esto fue corroborado por Korn et al, comparando controles con pacientes con esófago de Barrett ${ }^{6}$. La primera evaluación objetiva en pacientes con síntomas de reflujo gastroesofágico es la endoscopia alta, que permite evaluar la dilatación del cardias durante la manobria de retrovisión. Por lo tanto, la relación entre el endoscopio y el pliegue cardial puede ser claramente observada.

El objetivo del presente estudio fue comparar el aspecto endoscópico del cardias con los hallazgos de los estudios funcionales esofágicos, como es la presión de reposo del esfínter gastroesofágico, su competencia y la exposición al reflujo ácido patológico en un grupo de pacientes con síntomas de reflujo GE crónico.

\section{MATERIAL y MÉTOdos}

Pacientes. En este estudio prospectivo se incluyeron 150 pacientes con síntomas de reflujo gastroesofágico (pirosis o regurgitación de más de 3 años de duración). Se trataba de 89 mujeres y 61 hombres, con una edad promedio de 52,6 años (rango 19 a 72). Se excluyeron pacientes con esófago de Barrett extenso (más de $3 \mathrm{~cm}$ de mucosa columnar tapizando el esófago distal), con cirugía esofágica o gástrica previa, con complicaciones pépticas como úlcera esofágica 0 estenosis, pacientes con esclerodermia o con displasia o adenocarcinomas.

Análisis endoscópico. Este procedimiento se realizó en todos los pacientes después de un ayuno de $8 \mathrm{~h}$, con una premedicación consistente en anestesia tópica faríngea con dimecaína al $4 \%$ y administración intravenosa de midazolan y Buscapina ${ }^{\circledR}$. Todos los pacientes fueron monitorizados con oxímetro de pulso y todos los procedimientos se

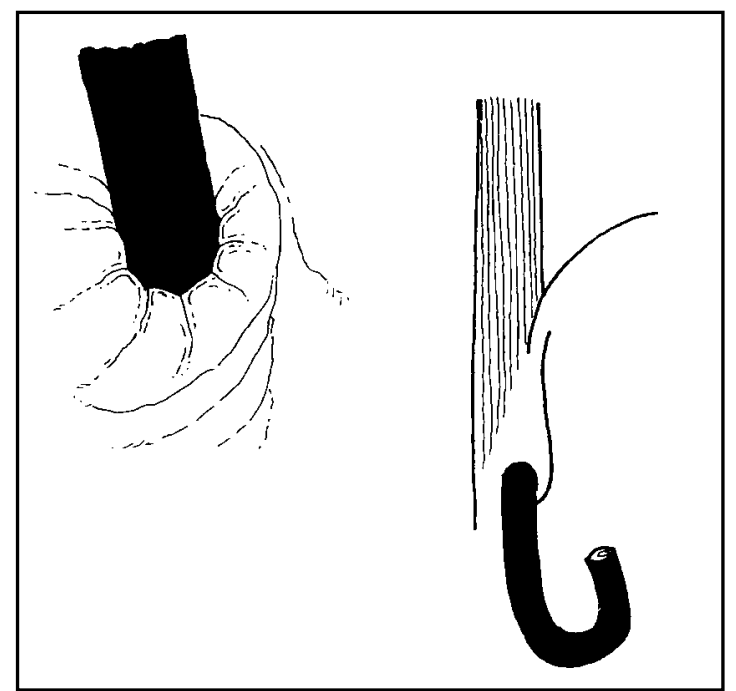

FiguRA 1. Representación esquemática del aspecto endoscópico del cardias durante retrovisión, mostrando un manguito adosado al endoscopio, con cardias cerrado que corresponde a cardias tipo I.

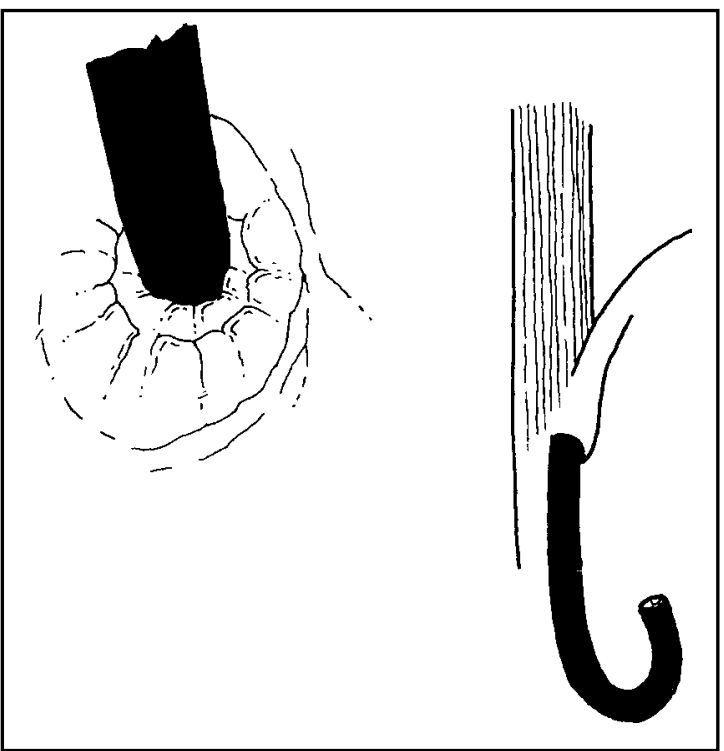

FiguRA 2. Aspecto endoscópico del cardias tipo II, en que las paredes de la unión gastroesofágica están adosadas al endoscopio en forma laxa, sin un manguito o anillo alrededor. 


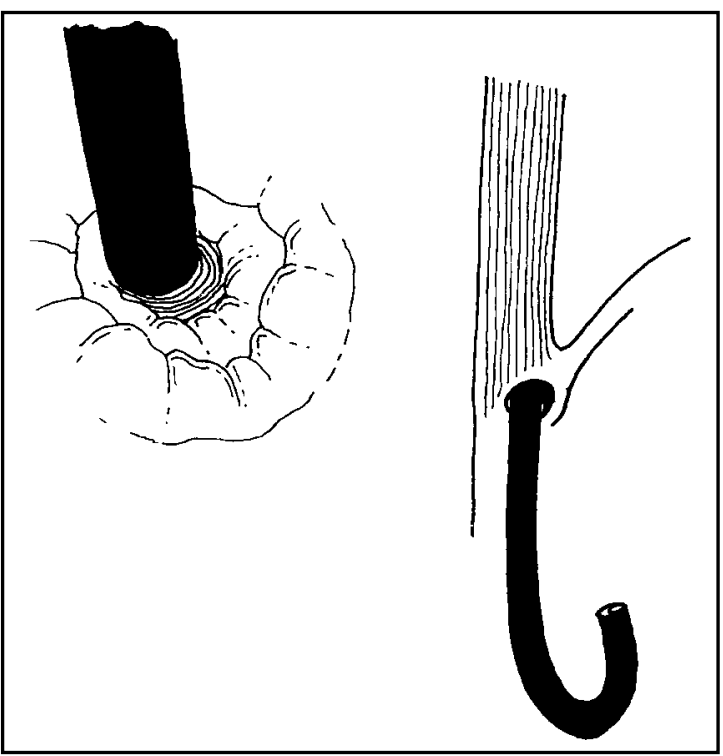

FIgURA 3. Aspecto endoscópico del cardias tipo III, en que hay un espacio entre las paredes de la unión gastroesofágica y el endoscopio.

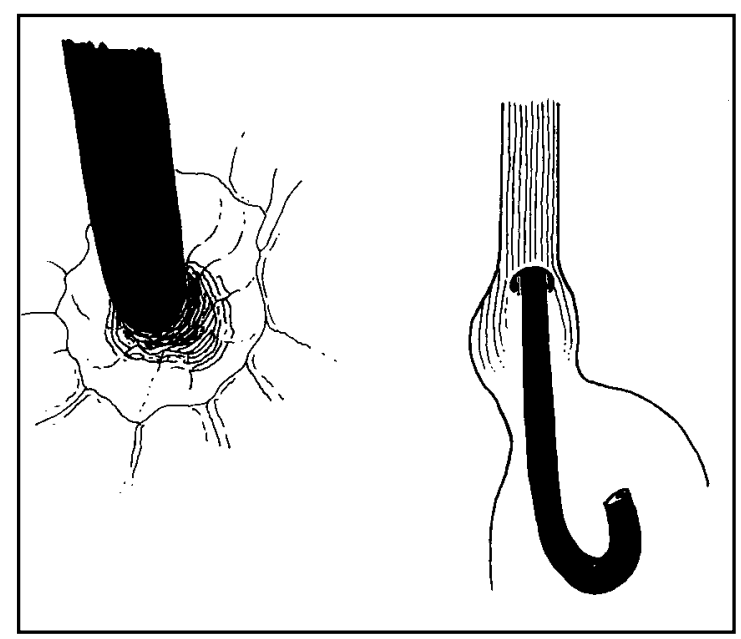

FIGURA 4. Aspecto endoscópico del cardias tipo IV, con una hernia hiatal y un amplio espacio entre las paredes de la unión gastroesofágica y el endoscopio. grabaron en video. El instrumento empleado fue un endoscopio GIFXQ-20 (Olympus, Japón). Para la clasificación endoscópica por retrovisión del aspecto del cardias se empleó la nomenclatura difundida por Hill et al ${ }^{7}$, que lo divide en 4 tipos:

a) Tipo I: presencia de un pliegue bien cerrado alrededor del endoscopio con 3 a $4 \mathrm{~cm}$ de extensión hacia la curvatura menor (hallazgo normal) (Figura 1). b) Tipo II: el pliegue es menor y durante la inspiración se forma un espacio entre el endoscopio y el cardias (Figura 2). c) Tipo III: casi no existe pliegue cardial y constantemente existe un espacio entre el endoscopio y el cardias mayor que el diámetro del instrumento (Figura 3). d) Tipo IV: presencia de una hernia hiatal y un importante espacio entre el endoscopio y el cardias (Figura 4).

Estudios manométricos. Todos los sujetos del presente estudio fueron sometidos a un análisis manométrico con la técnica descrita previamente ${ }^{8-}$ 10; empleando un sistema de 8 catéteres de polivinilo perfundidos mediante bomba neumohidráulica (Ardorfer Medical Specialities, Milwaukee). Los catéteres tienen 4 orificios, ubicados al mismo nivel y 4 orificios separados $5 \mathrm{~cm}$ entre sí, conectados a una computadora y analizados por un programa específico de motilidad esofágica (Synectics Medical, Suecia). La presión del esfínter gastroesofágico normal es $18 \pm 3 \mathrm{mmHg}$, y se consideró una presión menor que $10 \mathrm{mmHg}$ como una presión disminuida. Se consideró un EGE incompetente cuando cumplió al menos con una de las siguientes características: presión de reposo menor a $6 \mathrm{mmHg}$, largo total menor a $2 \mathrm{~cm}$ y largo abdominal menor a $10 \mathrm{~mm}^{11,12}$.

Monitoreo de pH intraesofágico de $24 \mathrm{~h}$. Este procedimiento se realizó inmediatamente después del estudio manométrico, una vez identificada la exacta ubicación del esfínter gastroesofágico. El catéter se coloca $5 \mathrm{~cm}$ por encima del límite proximal del esfínter gastroesofágico. Los detalles de esta técnica han sido publicados previamente ${ }^{13,14}$. La información se almacenó en un microcomputador portátil Digitrapper MKII (Synectics Medical, Suecia) y se analizó mediante un programa computacional (Gastrosoft, Dallas, Texas). Para el presente estudio se consideró una exposición ácida $(\mathrm{pH}<4)$ del esófago distal mayor al $4 \%$ durante las $24 \mathrm{~h}$ como un RGE patológico. 
Estadística. Para los cálculos estadísticos, los pacientes con cardias tipo I y II se consideraron como «cardias normal», los tipo III y IV como «cardias patológico o dilatado». Para los cálculos de significación estadística se empleó el test exacto de Fisher, tomando un $p<0,05$ como significativo. También se calculó la sensibilidad, la especificidad, el valor predictivo positivo (VPP) y el valor predictivo negativo (VPN) del cardias considerado normal o patológico en relación al $\mathrm{pH}$ de $24 \mathrm{~h}$.

\section{Resultados}

Los 150 pacientes se distribuyeron de la siguiente manera según el aspecto endoscópico del cardias: tipo I 30 pacientes, tipo II 16 pacientes, tipo III 75 pacientes y tipo IV 29 pacientes. Esto significa que los tipos I y II correspondieron a 46 pacientes $(30,6 \%)$ y los tipos III y IV a 104 pacientes $(69,3 \%)$. La Figura 5 muestra la distribución de los pacientes según sexo y tipo de cardias, no encontrando diferencias significativas en este aspecto. La Tabla 1 muestra los hallazgos endoscópicos en relación al tipo de cardias. Hubo una diferencia muy significativa en los hallazgos endoscópicos del esófago distal comparando ambos grupos. La presión del esfínter fue bajo $10 \mathrm{mmHg}$ en $67,3 \%$ de pacientes con cardias tipo III y IV, mientras que sólo $28,2 \%$ de los tipos I y II tuvieron una presión de reposo del EGE menor a $10 \mathrm{mmHg}$ ( $\mathrm{p}<0,001$ ) (Tabla 2). La Tabla 2 también muestra el porcentaje de pacientes con esfínter GE incompetente según el aspecto endoscópico del cardias, observando una diferencia significativa entre ambos grupos ( $p<0,001)$. El reflujo ácido

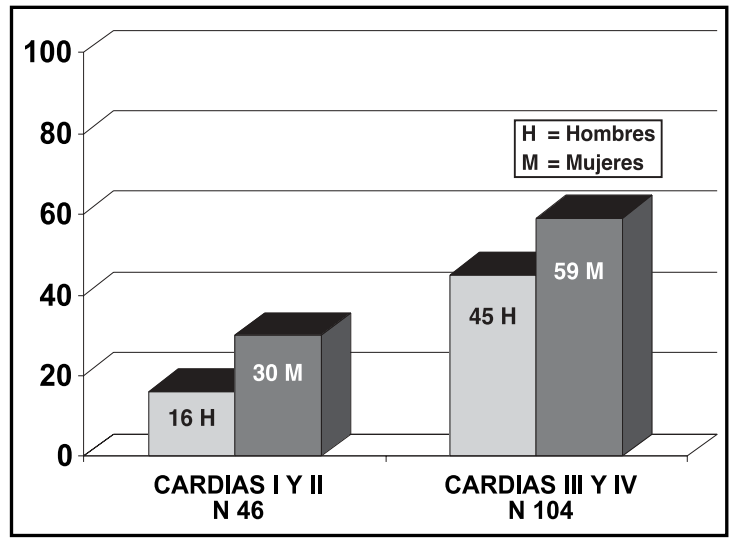

FiguRA 5. Distribución por género según el aspecto endoscópico del cardias en pacientes con síntomas de reflujo gastroesofágico.

patológico (Figura 6) estuvo presente en $43,4 \%$ de los pacientes con cardias tipo I y II y en $71,1 \%$ en los pacientes con cardias III y IV $(p<0,001)$. La clasificación endoscópica del cardias en normal» (tipos I y II) y «dilatado»(tipos III y IV) en relación a la presencia de reflujo ácido patológico obtuvo una sensibilidad de 78,7\%, una especificidad de $46,4 \%$, un VPP de $71,1 \%$ y un VPN de $56,5 \%$.

\section{DISCUSIÓN}

Los resultados del presente estudio sugieren que los pacientes con cardias endoscópicamente abierto o dilatado, tienen significativamente un esfínter GE más hipotensivo e incompetente que pacientes con cardias de tipo normal. Resultados

Tabla 1. H allazgo endoscópico en relación al tipo del cardias

\begin{tabular}{|lccc|}
\hline $\begin{array}{l}\text { Hallazgo endoscópico } \\
\text { del esófago }\end{array}$ & $\begin{array}{c}\text { I y II } \\
\mathrm{n}=46\end{array}$ & $\begin{array}{c}\text { Cardias tipo } \\
\text { III y IV } \\
\mathrm{n}=104\end{array}$ & Valor $\mathrm{p}$ \\
\hline Normal & $34(71,9 \%)$ & $23(22,1 \%)$ & $<0,001$ \\
Esofagitis & $4(8,6 \%)$ & $35(33,6 \%)$ & $<0,001$ \\
Barrett corto & $9(19,5 \%)$ & $58(56,7 \%)$ & $<0,001$ \\
Hernia hiatal & - & $33(31,7 \%)$ & $<0,001$ \\
\hline
\end{tabular}


Tabla 2. Presión EGE, largo abdominal EG E, largo total EGE, porcentaje EG E hipotenso, porcentaje incompetencia EGE en relación al tipo endoscópico de cardias

\begin{tabular}{|lccc|}
\hline & $\begin{array}{c}\text { Cardias normal } \\
\mathrm{n}=46\end{array}$ & $\begin{array}{c}\text { Cardias dilatado } \\
\mathrm{n}=104\end{array}$ & Valor $\mathrm{p}$ \\
\hline Presión EGE $\overline{\mathrm{x}} \pm \mathrm{DE}(\mathrm{mmHg})$ & $14,7 \pm 6,21$ & $8,7 \pm 4,16$ & $<0,001$ \\
Largo ABD. EGE $\overline{\mathrm{x}} \pm \mathrm{DE}(\mathrm{mm})$ & $7 \pm 4$ & $4 \pm 4$ & 0,12 \\
Largo total EGE $\overline{\mathrm{x}} \pm \mathrm{DE}$ & $36 \pm 8$ & $33 \pm 9$ & 0,10 \\
$\%$ presión EGE $<10 \mathrm{mmHg}$ & $28,2 \%$ & $67,3 \%$ & $<0,001$ \\
$\%$ incompetencia EGE & $43,4 \%$ & $78,8 \%$ & $<0,001$ \\
\hline
\end{tabular}

EGE = Esfínter gastroesofágico

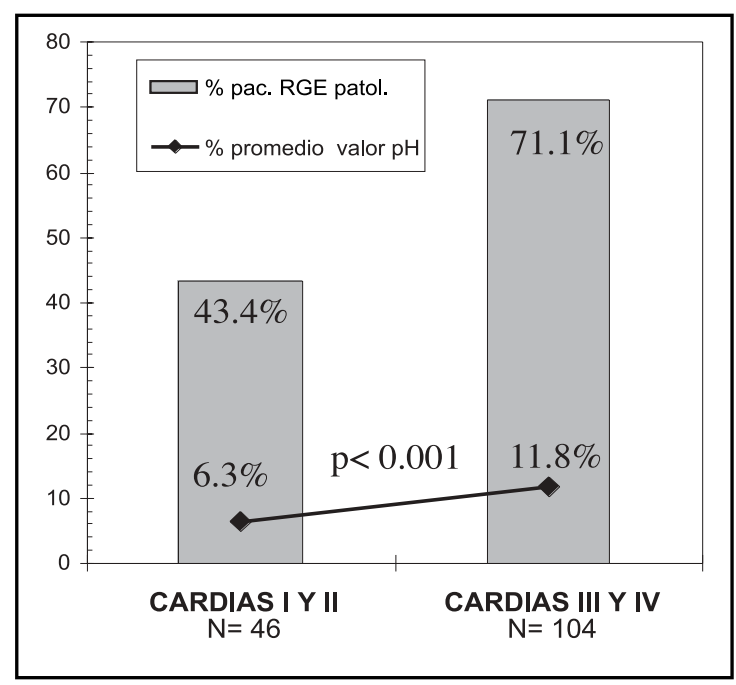

FiguRa 6. Porcentaje de pacientes con reflujo ácido patológico y valor promedio del porcentaje de tiempo con $\mathrm{pH}<4$ en $24 \mathrm{~h}$ en el esófago distal según aspecto endoscópico del cardias (6,3 y 11,3\%).

similares obtuvieron Oberg et $\mathrm{al}^{15}$. Nos parece que la evaluación de la presencia de un EGE incompetente es más completa y adecuada que la sola medición aislada de la presión de reposo esfinteriano, puesto que considera también el largo abdominal y el largo total del esfínter. Se puede apreciar en la Tabla 2 que casi la totalidad de los pacientes con cardias tipo III y IV tienen un EGE incompetente, pero llama la atención que $43,4 \%$ de los pacientes con cardias endoscópicamente normal también tienen un EGE incompetente. Esto sugiere que el hallazgo endoscópico de un cardias tipo I y II no excluye que el paciente pueda tener una válvula incompetente. Lo mismo vale para la medición de la exposición ácida del esófago distal durante $24 \mathrm{~h}$, en que $43,4 \%$ de los pacientes con cardias de tipo normal tienen reflujo GE patológico. Se conoce que un mecanismo muy importante para la presencia de RGE patológico es la ocurrencia frecuente de relajaciones transitorias del esfínter GE, teniendo una presión de reposo dentro de límites normales ${ }^{16}$. Por otro lado, en $29 \%$ de los pacientes con cardias dilatado no se detectó un reflujo ácido patológico. Este hallazgo sugiere que no fue capaz el método de detectar un reflujo patológico real ${ }^{17}$ o que algunos de estos pacientes pueden tener un reflujo no ácido. La pHmetría no detecta todos los eventos de reflujo GE, principalmente si poco 0 no ácido está presente en el material refluido ${ }^{17}$. Se ha descrito la presencia de reflujo no ácido o reflujo gaseoso, que no es medido por la pHmetría de $24 \mathrm{~h}$. Por eso se ha desarrollado el análisis de la impedancia intraluminal eléctrica que es capaz de monitorear el movimiento líquido o aéreo dentro del lumen esofágico ${ }^{18}$ y el Bilitec para medir el reflujo de contenido duode$\mathrm{nal}^{19}$, que hemos empleado con frecuencia. En resumen, el hallazgo endoscópico de un cardias «dilatado»sugiere que hay una alteración anatómica en estos pacientes con síntomas de RGE y que puede sugerir la presencia de un RGE patológico. Sin embargo, esta interpretación del aspecto endoscópico del cardias debe ser analizada con mucha cautela y no debe sugerir la necesidad de un tratamiento más radical y enérgico, como la cirugía, en ausencia de otros estudios funcionales del esófago, como la manometría computarizada y la pHmetría de $24 \mathrm{~h}$. 


\section{REFERENCIAS}

1. Cows JL Surgical control of reflux in hiatus hernia. Am J Surg 1988; 115: 465-71.

2. HIEBERT CA. Hiatal hernia, gastroesophageal reflux, and their complications. In Orringer $M B$, Zuidema GD (eds.): Shackelford's Surgery of the Alimentary Tract. Vol 1. The Esophagus. $3^{\text {rd }}$ ed. Saunders. Philadelphia, London, Toronto, Montreal, Sydney, Tokio, 1991; 164-75.

3. LARRAín A. Technical considerations in posterior gastropexy. Surg Gynec Obst 1971; 122: 299-300.

4. SKINNER DB. Pathophysiology of gastroesophageal reflux. Ann Surg 1985; 202: 546-56.

5. Csendes A, Miranda M, Espinoza M, Velasco N, Henriquez A. Perimeter and location of the muscular gastroesophageal junction or «cardia» in control subjects and in patients with reflux esophagitis or acalasia. Scand J Gastroent 1981; 16: 951-6.

6. Korn O, Csendes A, Burdiles P, Braghetto I, Stein HJ. Anatomic dilatation of the cardia and competence of the lower esophageal sphincter: a clinical and experimental study. J Gastroent Surg 2000; 4: 398-406.

7. Hill AD, Kozarek RA, Kraemer SJM, Aye RW, MERCER D, LOW DE, Pope CE II. The gastroesophageal flap valve: in vitro and in vivo observations. Gastrointest Endosc 1996; 44: 541-7.

8. Csendes A, Maluenda F, Braghetto I, Csendes P, Henriquez A, Queseda S. Location of the lower esophageal sphincter and the squamous columnar mucosal junction in 109 healthy controls and 778 patients with different degrees of endoscopic esophagitis. Gut 1993; 34: 21-9.

9. Csendes A, Coronel M, Avendaño R, Córdova $H$, ZeNTENo G. Localización endoscópica del cambio de mucosas escamoso-columnar en pacientes con diferentes grados de reflujo gastroesofágico patológico. Rev Méd Chile 1996; 124: 1320-4.
10. Csendes A, Braghetto I, Burdiles P, Díaz JC, Maluenda F, Korn O. A new physiological approach for the surgical treatment of patients with Barrett's esophagus. Ann Surg 1997; 226: 123-33.

11. Zaninotto G, DeMeEster TR, Schwizer W, Johansson $\mathrm{KE}, \mathrm{CHENG}$ SC. The lower esophageal sphincter in health and disease. Am J Surg 1988; 155: 104-11.

12. Csendes A, Burdiles P, Alvarez F, Maluenda F, HenríQuEZ A, QuesAdA S, CSENDES P. Manometric features of mechanically effective lower esophageal sphincter in controls and in patients with different degrees of gastroesophageal reflux. Dis Esoph 1996; 9: 290-4.

13. De Meester TR, Wang CI, Wemly JA. Technique, indications, and clinical use of 24-h intraesophageal $\mathrm{pH}$ monitoring. J Thorac Cardiovasc Surg 1980; 79: 656-70.

14. Csendes A, Alvarez F, Burdiles P, Braghetto I. Magnitud del reflujo ácido gastroesofágico medido por pHmetría de $24 \mathrm{~h}$ y manometría comparando el grado de esofagitis endoscópica. Rev Méd Chile 1994; 122: 59-67.

15. Oberg S, Peters JH, DeMeester tR, Lord RV, Johansson J, CRookes PF, Breemer CG. Endoscopic grading of the gastroesophageal valve in patients with symptoms of gastroesophageal reflux disease (GERD). Surg Endosc 1999; 13: 1184-8.

16. Dent J, Dodds WJ, Friedman RH, Sekiguchi T, Hogan WJ, Arndorfer RC ET al. Mechanism of gastroesophageal reflux in recurrent asymptomatic human subjects. J Clin Invest 1980; 65: 256-67.

17. Sifrim D, Castell D, Dent J, Kahrilas PJ. Gastroesophageal reflux monitoring: Review and consensus report on detection and definitions of acid, non-acid and gas reflux. Gut 2004; 53: 1024-31.

18. SiLWEY J. Intraluminal electrical impedance procedure for measurement of gastrointestinal motility. J Gastrointest Motil 1991; 3: 51-62.

19. BeCHI P, PuCCIANI F, BALDIN F. Long-term ambulatory enterogastric reflux monitoring validation of a new fiberoptic technique. Dig Dis Sci 1993; 38: 1297-306. 